\title{
BOUNDARY ELEMENT METHOD APPLIED TO ADDED MASS COEFFICIENT CALCULATION OF THE SKEWED MARINE PROPELLERS
}

Ehsan Yari, Ph. D.

Hassan Ghassemi, Prof.

Amirkabir University of Technology, Iran

\begin{abstract}
The paper mainly aims to study computation of added mass coefficients for marine propellers. A three-dimensional boundary element method (BEM) is developed to predict the propeller added mass and moment of inertia coefficients. Actually, only few experimental data sets are available as the validation reference. Here the method is validated with experimental measurements of the B-series marine propeller. The behavior of the added mass coefficients predicted based on variation of geometric and flow parameters of the propeller is calculated and analyzed. BEM is more accurate in obtaining added mass coefficients than other fast numerical methods. All added mass coefficients are nondimensionalized by fluid density, propeller diameter, and rotational velocity. The obtained results reveal that the diameter, expanded area ratio, and thickness have dominant influence on the increase of the added mass coefficients.
\end{abstract}

Keywords: Added mass coefficient, Boundary element method, Skew marine propeller

\section{INTRODUCTION}

In order to calculate the critical speed of a propeller shaft, it is necessary to know accurate values for the marine propeller added mass and added moment of inertia. In the case of a controllable pitch propeller, the values of the added mass and added moment of inertia are changed by varying the pitch angle, nondimensionalized by the mass and moment of inertia of the displaced fluid, respectively. It can be noted that the added moment of inertia is greatly influenced by the pitch angle. Calculating the added mass coefficient is difficult, especially for bodies of complex shapes. Generally, the added mass coefficient is a second order tensor that depends on the fluid acceleration vector through which the force vector on the body is obtained.

There are several publications discussing the added mass coefficients for marine propellers. Burrill and Robson (1962) conducted experiments to study the added mass of a series propeller [1]. Sahin et al. (1993) introduced the concept of added mass that generalized moving a desired object in different flow regimes [2]. Vernon et al. (1988) considered a surface panel method for calculation of added mass matrices, [3], an approach which is relatively close to the present study. Added mass estimations for a multi-component deeply submerged vehicle were studied by Watt [4]. Bermudez et al [5] investigated fluid-solid vibrations using finite element solution to derive the added mass formulation. Perrault et al. (2003) presented sensitivity of a typical AUV response to changes of hydrodynamic parameters. That analysis was performed using an axisymmetric computer model. The sensitivity of the added mass coefficients of a typical autonomous underwater vehicle (AUV) was presented as a response to changes of geometric parameters [6-7]. Koo and Lee (2003) developed a FAMD code to calculate the fluid added mass and damping of arbitrary structures submerged in confined viscous fluid [8]. Li et al. (2005) studied numerically the fluid flow and the added mass induced by vibration of a structure using the singularity distribution method in 0which axial and rotational motion of the structure was taken into account [9]. Wakaba \& Balachandar considered numerical simulations of the flow around a rigid sphere subjected to sudden acceleration (or deceleration) in a relative velocity [10]. Jianjun Long et al. reported estimation of the added mass and drag coefficient for a small remotely operated vehicle [11]. Chan \& Kang indicated that the drag and added mass coefficients are essential parameters in determining dynamic behavior of a submerged vessel [12]. The added mass coefficients of the Darpa Suboff submarine were calculated by means of the FMBEM [13]. Naveed Raza et al. determined the added mass by simulating the flow about a standard ellipsoid using a commercial CFD code [14]. Recently, a 3-D boundary element method was used by Gaschler and Abdel-Maksoud (2014) to compute the added mass coefficients for a marine propeller under cavitating condition [15]. 
In the present study, the boundary element method has been extended to enable calculation of the added mass matrix of skewed marine propellers. Then, appropriate grids generated on the geometry of marine propeller (blade, hub and boss) are investigated in order to provide correct results of added mass matrix coefficients. Finally, the added mass coefficients of a skewed marine propeller are presented as polynomial functions of selected geometric parameters.

\section{GOVERNING EQUATIONS}

Hydrodynamic forces and moments are determined by fluid inertia and viscous properties, according to the motion of a body in real incompressible fluid. Definitely, the other forces and moments can be calculated by obtaining either inertia or viscous terms. Since the fluid can be assumed inviscid, the inertia forces and moments can be calculated using this estimation. With the aid of this method, the forces and moments of inertia can be introduced as body added mass terms.

Let us consider the closed area $\Omega$, the boundary $S$, and the unit vector $\vec{n}$ normal to $S$ (Fig. 1). The boundary $S$ is composed of the wetted surface $S_{B}$ of the body, the wake surface $S_{W}$, and the external control surface $S_{\infty}$ encircling the surface areas $\mathrm{S}_{\mathrm{B}}$ and $\mathrm{S}_{\mathrm{W}}$.

$\Omega$ encounters the upstream flow of uniform velocity $\overrightarrow{\mathrm{V}}_{\mathrm{I}}$. The flow is assumed incompressible, inviscid and irrotational.

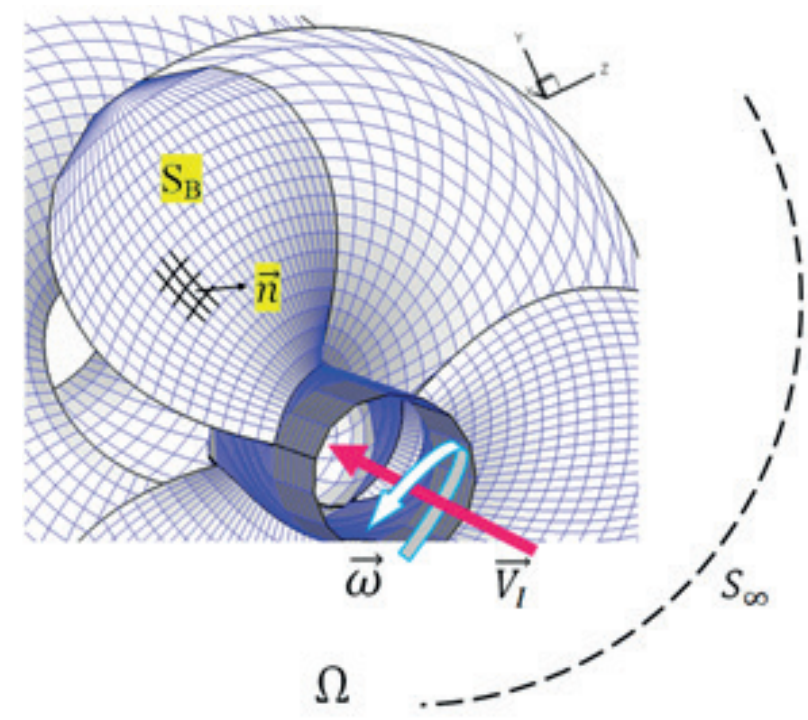

Fig. 1. Three-dimensional propeller

With the above assumptions, the flow field around the body can be identified using the perturbation velocity potential $\phi$ which satisfies the Laplace equation:

$$
\nabla^{2} \phi=0
$$

To solve the problem, the boundary conditions are considered as follows:

\section{BOUNDARY CONDITIONS}

Based on the kinematic boundary condition, the flow velocity normal to the body surface is equal to zero.

$$
\frac{\partial \Phi}{\partial n}=0 \stackrel{\text { yields }}{\longrightarrow} \frac{\partial \phi}{\partial n}=-V_{I} \cdot \boldsymbol{n}
$$

where $\mathrm{n}$, is the unit vector normal to the outside of the boundary. Considering the unit potential kinematics of the boundary condition, the vector is changed as follows:

$$
\left.\frac{\partial \phi_{j}}{\partial n}\right|_{j=1 \rightarrow 6}=\left\{\begin{array}{c}
\left.n_{\boldsymbol{i}}\right|_{i=1 \rightarrow 3} ^{j=1 \rightarrow 3} \\
\left.\left(\boldsymbol{r} \times n_{i}\right)\right|_{i=1 \rightarrow 3} ^{j=4 \rightarrow 6}
\end{array}\right.
$$
[16].

where, $r$ is the vertical distance from the fixed origin point

At the boundary surface at infinity $S_{\infty}$, the perturbation velocity due to the body surface tends to zero.

$$
x \longrightarrow \infty \Rightarrow \nabla \phi \longrightarrow 0
$$

\section{WAKE BOUNDARY CONDITIONS}

Based on these conditions, there is no flow velocity jump on the wake vortex, however there is a velocity jump of the potential on the surface which equals the circulation $\Gamma$ around the fin. The mathematical relations between flow velocity and potential are expressed as follows:

$$
\left\{\begin{array}{c}
(\Delta \phi)_{S_{W}}=P^{B}-P^{F}=-\Gamma \\
\left(\Delta \frac{\partial \phi}{\partial \mathrm{n}}\right)_{S_{W}}=\left(\frac{\partial \phi}{\partial \mathrm{n}}\right)^{\mathrm{B}}-\left(\frac{\partial \phi}{\partial \mathrm{n}}\right)^{\mathrm{F}}=0
\end{array}\right.
$$

where $B$ and $F$ represent the back and face side of the propeller, respectively [17].

\section{KUTTA CONDITION}

Hess and Smith showed that the flow passing along a thin and non lifting body can be described by distribution of source singularities. But to describe the flow passing along a lifting body, modeled by circulation distribution on the surface, a boundary condition for the trailing edge of the body, expressing that the velocity at the trailing edge should be limited and unique, is to added [18].

$$
|\nabla \phi|<0
$$

\section{ADDED MASS FORMULATION}

The added mass and added moment coefficients can be formulated using the force point of view and the kinetic 
energy theory. The added mass formulation is given by Eq (7). According to this equation, the added mass and added moment coefficients are only functions of body geometry.

$$
\lambda_{i k}=-\rho \int_{S} \frac{\partial \phi_{i}}{\partial n} \phi_{k} d S
$$

$\lambda_{\mathrm{ik}}$ is called the added mass of the body. The added mass matrix flowchart of the propeller for which the solving process will be continued until the results are converged to an accurate point is shown in Figure 2. [19]

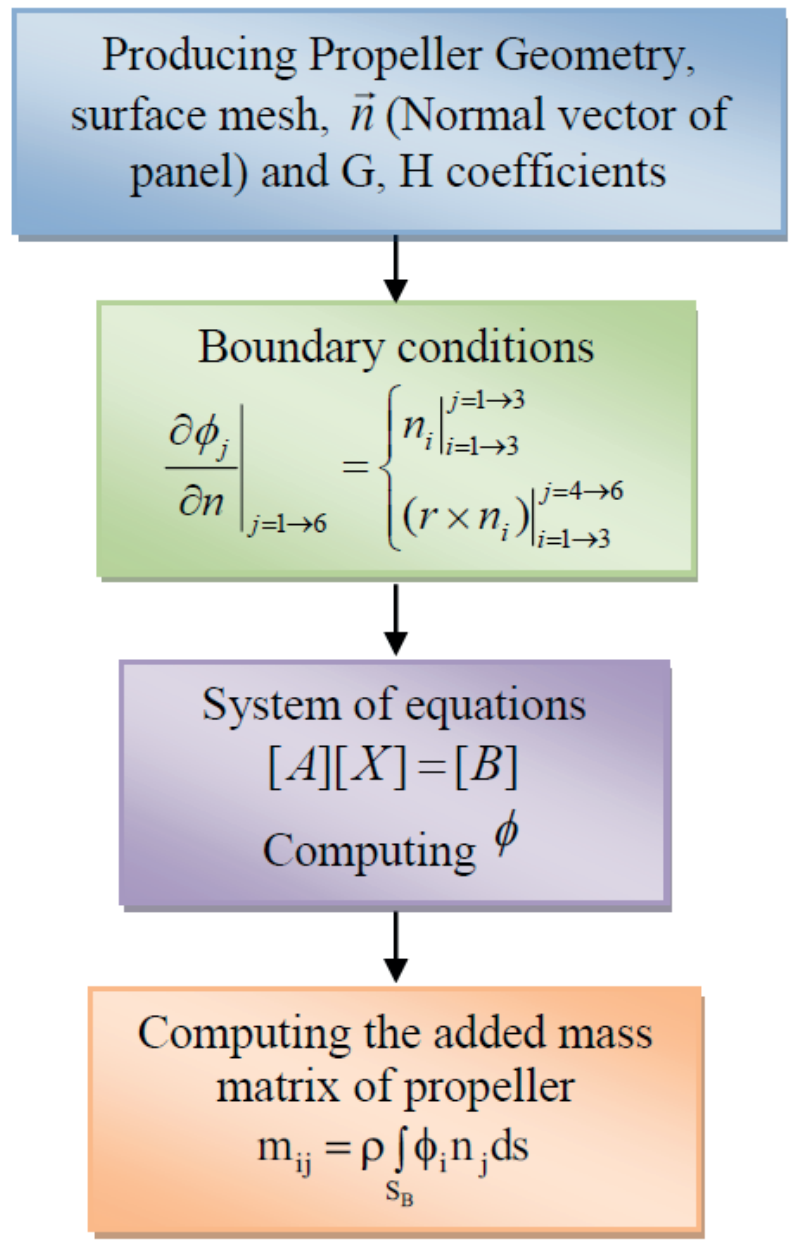

Fig. 2. Flowchart of the added mass matrix of the propeller

\section{BOUNDARY INTEGRAL EQUATION}

Using Green's theory, the general solution to the Laplace equation for each point $\mathrm{P}$ can be expressed as the following integral equation:

$$
\begin{aligned}
& 2 \pi \phi_{p}=\iint_{S_{B}}\left[\phi_{q} \frac{\partial}{\partial n_{q}}\left(\frac{1}{R_{p, q}}\right)-\right. \\
& \left.\frac{\partial \phi}{\partial n_{q}}\left(\frac{1}{R_{p, q}}\right)\right] d S+\iint_{S_{W}}\left[\Delta \phi_{q} \frac{\partial}{\partial n_{q}}\left(\frac{1}{R_{p, q}}\right)\right] d S
\end{aligned}
$$

where the subscripts $\mathrm{p}, \mathrm{q}$ correspond to the control and variable points in the integration, respectively. In the above equation, $\partial \phi / \partial \mathrm{n}$ is determined using the boundary condition of Eq. (3). $1 / R(p, q)$ is the distance between p and q, so the only remaining unknown for solving Eq. (8) is $\phi$.

\section{DISCRETIZATION OF EQUATIONS}

In order to solve Eq. (8), the equations are discretized and the body boundary surface is meshed with tetragonal elements. Fractional equations lead to a linear system of algebraic equations for $\phi$ and $i$, as follows:

$$
\begin{aligned}
2 \pi \phi_{i}=\sum_{j=1}^{N} D_{i j} & \left(\phi_{i}\right)+\sum_{j=1}^{N_{R}} \sum_{l=1}^{N_{W}} W_{i j l}(\Delta \phi)_{\mathrm{i}} \\
& +\sum_{\substack{j=1\\
}}^{N} S_{i j}\left(\frac{\partial \phi}{\partial n}\right)_{j} i \\
& =1,2, \ldots, N_{\text {total }}
\end{aligned}
$$

where $\mathrm{D}_{\mathrm{ij}}, \mathrm{S}_{\mathrm{ij}}$ and $\mathrm{W}_{\mathrm{ijl}}$ are the dipole and source coefficients for the $j$-th element that acts on the arithmetic point $i$. These dipole and source coefficients are defined as follows:

$$
\begin{aligned}
& D_{i j}=\sum_{k=1}^{k}\left[\iint_{S_{B}}\left[\frac{\partial}{\partial n_{j}}\left(\frac{1}{R_{i j}}\right) d S_{j}\right]_{k} \text { on } S_{B}\right. \\
& W_{i j l}=\sum_{k=1}^{k}\left[\iint_{S_{W}}\left[\frac{\partial}{\partial n_{j}}\left(\frac{1}{R_{i j}}\right) d S_{j}\right]_{k} \text { on } S_{W}\right. \\
& S_{i j}=\sum_{k=1}^{k}\left[\iint_{S_{B}}\left[\left(\frac{1}{R_{i j}}\right) d S_{j}\right]_{k} \text { on } S_{B}\right.
\end{aligned}
$$

To obtain these coefficients, numerical solution of the integrals is employed. For this purpose the following matrix equation is formed and then solved using the Gauss - Seidel method, leading to $\phi_{j}$ values [20].

$$
[\mathrm{D}][\phi]=[\mathrm{S}]+[\mathrm{W}][\Delta \phi]
$$

where $[\mathrm{D}]=$ dipole potential matrix, $[\mathrm{S}]=$ source potential matrix, and $[\mathrm{W}]=$ dipole potential matrix (Wake).

For lifting and non-lifting bodies, the use of the above computational method only differs by application, or not, of the Kutta boundary condition. The results are obtained directly regarding the kinematic boundary condition.

Although the Kutta condition is considered to analyze the propeller, the course of obtaining the results reveals no significant difference, as compared to cases without considering the Kutta condition. Therefore, regarding this remark, only D and S matrixes in equation 11 need to be determined.

$$
\begin{aligned}
& D_{i j}=\sum_{k=1}^{k}\left[\iint_{S_{B}}\left[\frac{\partial}{\partial n_{j}}\left(\frac{1}{R_{i j}}\right) d S_{j}\right]_{k} \text { on } S_{B}\right. \\
& S_{i j}=\sum_{k=1}^{k}\left[\iint_{S_{B}}\left(\frac{1}{R_{i j}}\right) d S_{j}\right]_{k} \text { on } S_{B}
\end{aligned}
$$


After extracting D and S, the integrals in Eq. (12), being in fact the same as general terms in Eq. (8), take the form:

$$
\begin{gathered}
\int_{S_{B}}\left[\frac{1}{R} \frac{\partial \phi}{\partial n}\right] d S=\left.\sum_{\substack{k=1 \\
k \neq i}}^{N} \frac{1}{R} \frac{\partial \phi_{j}}{\partial n}\right|_{j=1 \rightarrow 6} \delta S_{k} \\
\int_{S_{B}}\left[\phi \frac{\partial}{\partial n}\left(\frac{1}{R}\right)\right] d S=\sum_{\substack{k=1 \\
k \neq i}}^{N}\left[\frac{\partial}{\partial n}\left(\frac{1}{R}\right)\right] \delta S_{k}+2 \pi \phi_{i=k}
\end{gathered}
$$

If the above integrals (Eq.13) are formulated based on matrix equations, the following relation is derived:

$$
\left[\mathrm{A}_{\mathrm{ik}}\right]\left[\phi_{\mathrm{k}}\right]=\left[\mathrm{B}_{\mathrm{i}}\right]
$$

So that $\mathrm{i}=\mathrm{K}$ and $\mathrm{A}_{\mathrm{ik}}=2 \pi$

The added mass matrix values obtained by solving the matrix equations for all six potential unit functions can be defined as follows:

$$
\lambda_{i k}=-\rho \int_{S_{B}} \phi_{i} n_{k} d S_{B}
$$

If the above equation is written in the matrix form, which is the general form of the added mass matrix, the following matrix is introduced [14].

$$
\begin{aligned}
& \text { Added Mass Matrix }= \\
& {\left[\begin{array}{ccc}
\sum_{i=1}^{N} \phi_{1}^{i} n_{1}^{i} \delta S_{i} & \cdots & \sum_{i=1}^{N} \phi_{1}^{i}(r \times n)_{3}^{i} \delta S_{i} \\
\vdots & \ddots & \vdots \\
\sum_{i=1}^{N} \phi_{6}^{i} n_{1}^{i} \delta S_{i} & \cdots & \sum_{i=1}^{N} \phi_{6}^{i}(r \times n)_{3}^{i} \delta S_{i}
\end{array}\right]}
\end{aligned}
$$

\section{ADDED MASS OF B-SERIES PROPELLER}

In this section the added mass coefficients for a standard propeller are studied. The dimensions of the B-Series propeller selected for this study are given in Table 1.

\begin{tabular}{l|c}
\multicolumn{2}{c}{ Tab. 1. Main dimensions of the propeller } \\
\hline \multicolumn{1}{c}{ Propeller Type } & B-series \\
\hline Diameter [m] & 1 \\
\hline Pitch Ratio & 0.8 \\
\hline Expanded Area Ratio & 0.6 \\
\hline Number of Blades & 4 \\
\hline Rake Distribution & 0.0 \\
\hline Skew Distribution (deg.) & 17.7 \\
\hline Hub Ratio & 0.2 \\
\hline Rotation & R.H \\
\hline Total Surface Element & 6900 \\
\hline
\end{tabular}

Since the added mass coefficients of the B-Series propeller are available [21], this part of analysis aims at validating the results computed by the boundary element code. The mesh independency of the numerical results was investigated, showing very small error in comparison with the existing data. The added mass matrix obtained from the numerical analysis for the B-series propeller is shown in Table 2 .

Tab. 2. Matrix of Added Mass coefficients per density for B-series propeller $\left(\times 10^{4}\right)$

\begin{tabular}{c|c|c|c|c|c}
\hline 664.9 & -0.2 & 0.1 & -86 & 0 & 0 \\
\hline-0.2 & 89.8 & 94.9 & 0 & 40.4 & 3.5 \\
\hline 0.1 & 94.9 & 89.7 & 0 & -3.5 & 40.4 \\
\hline-86 & 0 & 0 & 11 & 0 & 0 \\
\hline 0 & 40.4 & -3.5 & 0 & 29.3 & 0 \\
\hline 0 & 3.5 & 40.4 & 0 & 0 & 29.4 \\
\hline
\end{tabular}

In Fig. 3, the grid independency is evaluated for coefficients M11 and M44. As it can be seen, at 6900 surface elements the coefficient variations tend to zero.

Afterwards, the data obtained from the present study were validated by comparing with the experimental data [21], see Tab. 3. According to these results, for all three coefficients the maximum error percentage is of the order of $3.7 \%$.

Comparing the results of the numerical boundary element based analysis with the experimental data [21] for added mass coefficients of B-series propeller reveals good conformity between the numerical and experimental data.

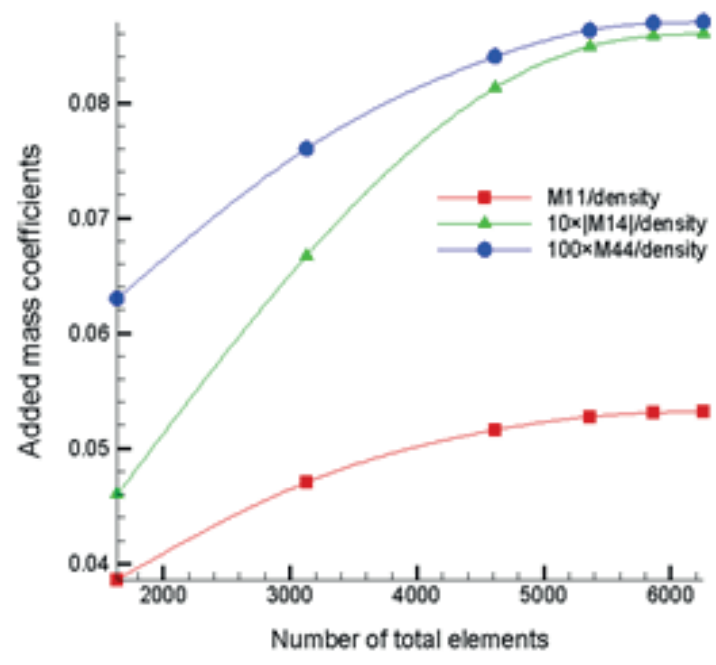

Fig. 3. Mesh independency study for $M_{11}$ and $M_{44}$

Tab. 3. Comparing numerical and experimental results for B-series propeller

\begin{tabular}{l|ccc}
\hline & $\mathbf{M}_{1 \mathbf{1 1}}$ & $\mathbf{M}_{\mathbf{1 4}}$ & $\mathbf{M}_{\mathbf{4 4}}$ \\
\hline Exp & 0.06906 & -0.00878 & 0.00111 \\
\hline Num & 0.06649 & -0.00860 & 0.00110 \\
\hline Difference & 0.00257 & -0.00018 & 0.00001 \\
\hline \% Error & 3.721402 & 2.050114 & 0.900901 \\
\hline
\end{tabular}




\section{COMPUTING THE ADDED MASS MATRIX FOR THE BENCHMARK PROPELLER}

According to the data and results obtained for the added mass matrix of the B-series propeller it can be found that there is a good accuracy between the experimental data [21] and the numerical solution. This section discusses computing the added mass matrix for a high skew propeller (HSP-4 Blades). Since the added mass matrix is only a function of the body geometry, the object of the study was the effects of changing geometrical parameters of the propeller. Firstly, the existing geometrical data of the propeller, based on the standards, were used to model the propeller geometry with very high accuracy. Then, the propeller surface and the hub were analyzed with respect to flow conditions. The propeller analysis was performed for different numbers of surface elements to arrive at the most appropriate variant for which the obtained answers did not depend on the number of elements.

Grid generation on the marine propeller is one of most important topics in extracting the accurate added mass coefficient matrix using the boundary element method. Since the propeller has the same number of zones as the

number of blades, the grid generated on each of these four sections is to be similar. It is noteworthy, however, that using a pave mesh on the propeller and especially on the hub may lead to incorrect results.

As it is shown in Figure 4, the grid was generated on the blades, hub, and boss, taking into account the above mentioned remarks.

Thereafter, the added mass matrix was derived for three cases: only blades, blades and middle hub, and finally complete geometry of the propeller. The effect of each geometric element was evaluated based on the obtained data.

According to the numerical results, each increase of the added mass coefficient rate amounted to less than one percent, regarding the middle hub with blades. When the boss was taken into account, the added mass coefficient rate increased significantly, by about at least 5 to 6 percent.

Thus, the geometric design of the boss has an important role in propeller design. In particular for high diameter propellers, optimizing the boss geometry is essential.

Tab. 4. Added mass matrix for HSP-4 blade propeller in three conditions

$\left[\begin{array}{cccccc}50.17 & 0.00 & 0.00 & -6.64 & 0.00 & 0.00 \\ 0.00 & 68.10 & -11.36 & 0.02 & 2.05 & -5.46 \\ 0.00 & -11.36 & 73.93 & -0.01 & 6.87 & 3.91 \\ -6.64 & 0.02 & -0.01 & 7.55 & 0.00 & 0.00 \\ 0.00 & 2.05 & 6.87 & 0.00 & 9.54 & 0.11 \\ 0.00 & -5.46 & 3.91 & 0.00 & 0.11 & 9.29\end{array}\right]$

Since the data compared to the main diameter are symmetrical and some coefficients have zero with accuracy of up to 5 decimal points, this testifies to high precision of the reported calculations (Tab. 4). The geometric and flow data of the high skew four-blade propeller are given in Tab. 5.
Tab. 5. Geometric and flow data of HSP-4 blade Propeller

\begin{tabular}{l|c}
\hline \multicolumn{1}{c|}{ Parameter } & Value \\
\hline Type & FPP \\
\hline No. of Blades, $Z$ & 4 \\
\hline Skew at $\mathrm{r} / \mathrm{R}=0.7$ & $41.62 \mathrm{deg}$ \\
\hline Hub Ratio, H/D & 0.20 \\
\hline Pitch Ratio, P/D & 0.80 \\
\hline Expanded Area Ratio & 0.60 \\
\hline Advance Ratio, J & 0.50 \\
\hline Diameter, D $(\mathrm{m})$ & 2 \\
\hline
\end{tabular}

\section{PARAMETERS AFFECTING THE ADDED MASS OF THE SKEWED PROPELLER}

Since the added mass coefficient is only a function of propeller geometry, its changes have been studied based on the sensitivity to geometrical parameters.

Figure 4-a shows the calculated variations of predominant added mass matrix coefficients as the propeller diameter increases. As can be seen, for most coefficients these variations have the form of the polynomial of the order of 3 . Only the coefficients $\mathrm{M}_{44}$ and $\mathrm{M}_{11}$ form the polynomials of the order of 2 and 4, respectively. These changes are quite noticeable in the case of $\mathrm{M}_{11}$ and the 2-order polynomial, while $\mathrm{M}_{44}$ can also be considered as the approximation by the 3-order polynomial. Consequently, it can be concluded that changes of most general mass matrix coefficients have the form of the 3 -order polynomial as the propeller diameter increases.

Figure 4-b shows changes of the added mass matrix coefficients in response to changes of the expanded area ratio of the propeller. According to the obtained results, it can be found that the changes of all moment and added mass coefficients are of the order of 2 as the expanded area ratio of the propeller increases.

Figure 5-c shows changes of the added mass coefficients as functions of the maximum thickness of the propeller profile section. For all diagonal coefficients this relation is linear, while for other coefficients it has the form on a 3-order polynomial.

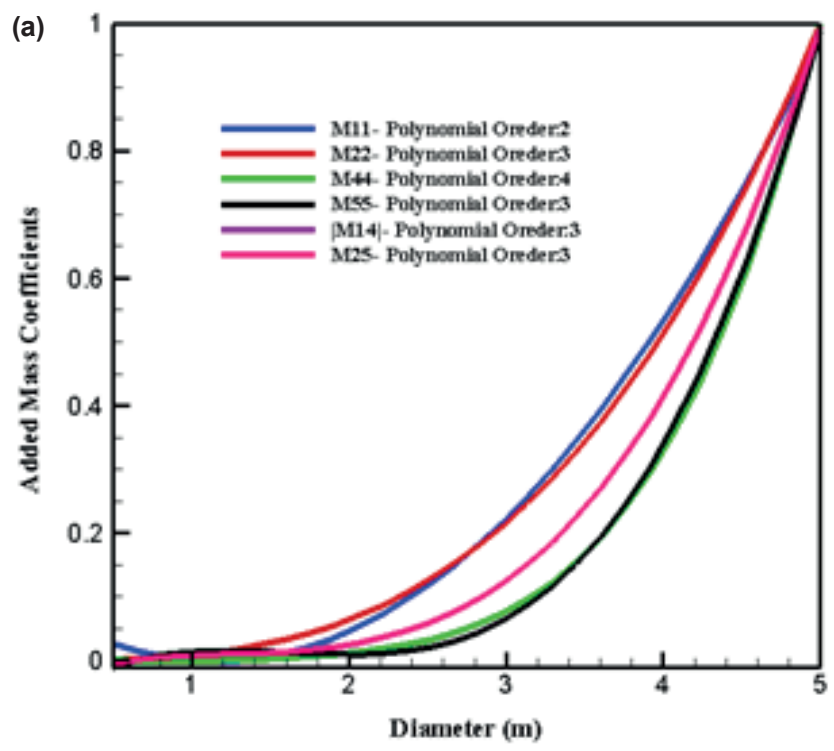

POLISH MARITIME RESEARCH, No 2/2016 

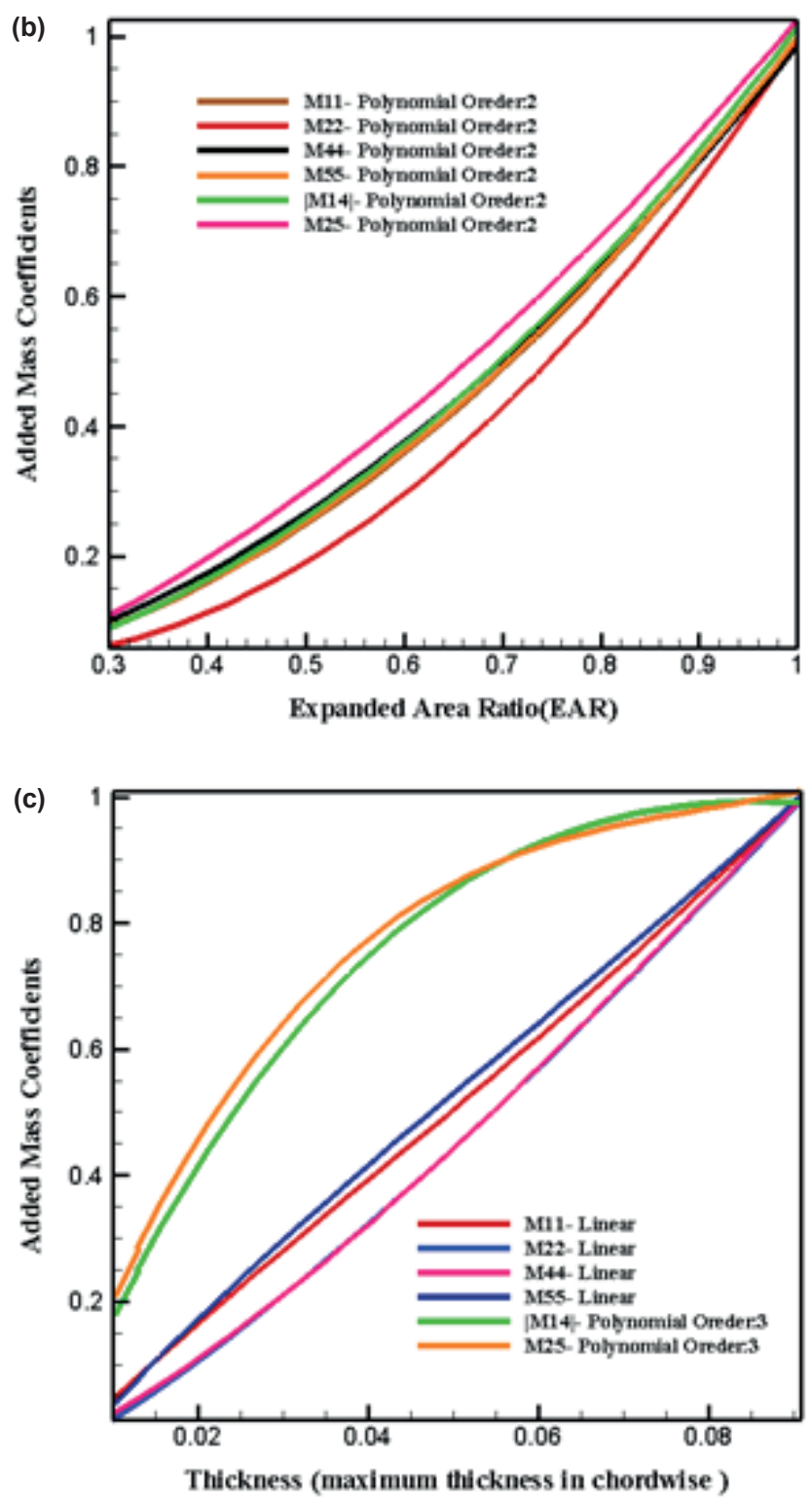

Fig. 4. Changes of the added mass coefficients as functions of geometrics parameters of the propeller

\section{CONCLUSIONS}

All coefficients cannot be derived using the reference formulation. However, they can be obtained with appropriate accuracy using the present study. As mentioned in the article, the added mass matrix of the $B$-series propeller was extracted and the results were validated with the available data.

The largest obtained error, compared to the experimental data, was about 3.7 percent, which is a very small amount, and for other coefficients the error rate was even fairy smaller.

Therefore, all the added mass and added moment coefficients can be calculated with good accuracy using the present study and taking into account the earlier mentioned remarks about propeller geometry generation.

Afterwards, the added mass matrix for a benchmark skewed propeller in various geometrical data was computed. According to the obtained results, changes of the added mass coefficients of the propeller caused by varying geometrical parameters such as diameter, expanded area ratio, and thickness, had the form of polynomial function of cubic, quadratic, and linear and quadratic order (3-2-1-2), respectively.

\section{REFERENCES}

1. Burrill, L. C., Robson, W.: Virtual mass and moment of inertia of propellers. Trans. North East Coast Institution of Engineers and Shipbuilders 78, 325-360, 1962.

2. Sahin I, Crane JW and Waston KP: Added mass coefficients for submerged bodies by a low-order panel method. J Fluids Eng 1993; 115:452-7.

3. Sahin I, Crane JW, Waston KP: Application of a panel method to hydrodynamics of underwater vehicles. Ocean Eng 1997; 24(6):501-12.

4. George D. Watt: Estimates for the added mass of a multicomponent, deeply submerged vehicle. Defense Research Establishment Atlantic (Report), October 1988.

5. Bermudez Alfredo, Rodr'iguez Rodolfo, Santa Marina Duarte: A finite element solution of an added mass formulation for coupled fluid-solid vibrations. Numer. Math. (2000) 87: 201-227

6. Perrault Doug, Bose Neil, O'Young Siu, Williams D. Christopher: Sensitivity of AUV response to variations in hydrodynamic parameters. Ocean Engineering 30 (2003) 779-811.

7. Perrault Doug, Bose Neil, O'Young Siu, and Williams D. Christopher: Sensitivity of AUV added mass coefficients to variations in hull and control plane geometry. Ocean Engineering 30 (2003) 645-671.

8. Gyeong-Hoi Koo, Jae-Han Lee: Development of FAMD Code to Calculate the Fluid Added Mass and Damping of Arbitrary Structures Submerged in Confined Viscous Fluid. KSME International Journal, Vo£ 17 No. 3, pp. 457 466 2003.

9. Su Li, Li Shu-Juan, and Tan Guo-an: Numerical analysis of Fluid Flow and Added Mass Induced by Vibration of Structure. Applied Mathematics and Mechanics, Vol. 26, No 2, Feb 2005.

10. Wakaba L. S S. Balachandar: On the added mass force at finite Reynolds and acceleration numbers. Theor Comput Fluid Dyn. (2007) 21: 147-153.

11. Jianjun Long, Baihai Wu, Jinping Wu, Tibing Xiao and Lili Wang: Estimation of Added Mass and Drag Coefficient for a Small Remotely Operated Vehicle. Proceedings of the 2008 IEEE International Conference on Information and 
CONTACT WITH THE AUTHORS

12. Wai Leung Chan and Taesam Kang: Simultaneous Determination of Drag Coefficient and Added Mass. IEEE Journal of Oceanic Engineering, Vol. 36, No. 3, July 2011.

13. Zhiliang Lin, Shijun Liao: Calculation of added mass coefficients of 3D complicated underwater bodies by FMBEM “,Commun Nonlinear Sci Numer Simulat 16 (2011) 187-194.

14. Naveed Raza, Imran Mehmood, Hafiz Rafiuddin and Mohammad Rafique: Numerical Simulation of Added Mass Determination of Standard Ellipsoids. Proceedings of 20129 th International Bhurban Conference on Applied Sciences \& Technology (IBCAST).

15. Gaschler, M., Abdel-Maksoud, M.: Computation of hydrodynamic mass and damping coefficients for a cavitating marine propeller flow using a panel method. Journal of Fluids and Structures 49, 574-593, 2014.

16. Olivier Saout: Computation of Hydrodynamic Coefficients and Determination of Dynamic Stability Characteristics of an Underwater Vehicle Including Free Surface Effects. Ms Thesis, Florida Atlantic University, May 2003.

17. Kinnas S.A. and Hsin C.Y: Boundary Element Method for the Analysis of the Unsteady Flow around Extreme Propeller Geometry. AIAA, Journal, 30 (3), 1992.

18. Hess J T, Smith A M: Calculation of non-lifting potential flow about arbitrary three-dimensional bodies. Journal of Ship Research,8(2),1964.

19. Ghassemi H, Yari E.: The Added Mass Coefficient computation of sphere, ellipsoid and marine propellers using Boundary Element Method. Polish Maritime Research 1(68) 2011 Vol 18; pp. 17-26.

20. Morino L and Kuo C.C: Subsonic Potential Aerodynamics for Complex Configuration: A general Theory. AIAA Journal, 12 (2), 191-197, 1974.

21. Alexandr I. Korotkin: Added mass of ship structure. Fluid mechanics and its applications, Volume 88, 2009.
Ehsan Yari

Faculty of Maritime Engineering Amirkabir University of Technology Hafez Ave., Tehran, IRAN

E-mail: ehsanyari_mechanical@yahoo.com

\section{Hassan Ghassemi}

Faculty of Maritime Engineering, Amirkabir University of Technology Hafez Ave., Tehran, IRAN

E-mail: gasemi@aut.ac.ir 